\title{
Downregulation of $14-3-3 \sigma$ in ovary, prostate and endometrial carcinomas is associated with CpG island methylation
}

\author{
Paulette Mhawech ${ }^{1}$, Ana Benz ${ }^{1}$, Christelle Cerato ${ }^{1}$, Vincent Greloz ${ }^{1}$, Mounia Assaly ${ }^{1}$, \\ Julian C Desmond ${ }^{2}$, H Phillip Koeffler ${ }^{2}$, Dimitri Lodygin ${ }^{3}$, Heiko Hermeking ${ }^{3}$, \\ François Herrmann ${ }^{4}$ and Juerg Schwaller ${ }^{1}$ \\ ${ }^{1}$ Department of Clinical Pathology, Geneva University Hospital, Geneva, Switzerland; ${ }^{2}$ Division of \\ Hematology-Oncology, Cedars Sinai Medical Center, UCLA, Los Angeles, USA; ${ }^{3}$ Division of Molecular \\ Oncology, Max Planck-Institute of Biochemistry, Martinsried, Germany and ${ }^{4}$ Rehabilitation and Geriatrics, \\ Geneva University Hospital, Geneva, Switzerland
}

\begin{abstract}
The 14-3-3 $\sigma$ inhibitor of cell cycle progression has been shown to be target of epigenetic deregulation in many forms of human cancers; however, its role in urological and gynecological cancers has not been studied. Here, we have analyzed the expression of 14-3-3 $\sigma$, wild-type p53 and mutated p53 in over 300 cases of the most common cancers occurring in the urological and gynecological tracts and its normal counterpart tissue by immunohistochemistry using the multiple tumor tissue microarrays. 14-3-3 $\sigma$ expression was detected in normal epithelia from most organs with sporadic expression in renal tubules and absence in the testis. In contrast to normal tissue, 14-3-3 $\sigma$ expression was lost in $40-60 \%$ of adenocarcinomas of the breast, ovary, endometrium and prostate. There was no association between 14-3-3 $\sigma$ and wild-type/mutated p53 expression. By performing methylation-specific PCR, we showed a close association of 14-3-3 $\sigma$ CpG island methylation and low protein expression levels of 14-3-3 $\sigma$. In addition, a direct link of 14-3-3 $\sigma$ mRNA expression levels to CpG island methylation is demonstrated in two human cancer cell lines. Loss of 14-3-3 $\sigma$ expression due to promoter hypermethylation may represent the most frequent molecular aberration in ovarian, endometrial and prostate adenocarcinomas.
\end{abstract}

Modern Pathology (2005) 18, 340-348, advance online publication, 16 July 2004; doi:10.1038/modpathol.3800240

Keywords: urological and gynecological carcinomas; 14-3-3 $\sigma$ expression; CpG methylation

The 14-3-3 $\sigma$ protein belongs to a highly conserved family of acidic protein, the 14-3-3 family, which is constituted of at least seven mammalian isoforms. ${ }^{1-3}$ From all seven isoforms, $14-3-3 \sigma$ has been most directly linked to cancer. 14-3-3 proteins are defined as phosphoserine-binding proteins that can bind to the two consensus motifs RSXpSXP and RXY/ FXpSXP, which are present in almost all of the 143-3-binding proteins. 14-3-3 proteins are involved in regulation of numerous cellular signaling pathways and one of their main activities is cell cycle regulation. A functional link between 14-3-3 $\sigma$ and the p53 protein has been proposed. Upon DNA damage, p53 induces $14-3-3 \sigma$ expression. ${ }^{4}$ By

Correspondence: Dr P Mhawech, MD, Division of Clinical Pathology, Geneva University Hospital, CMU, 1 Rue Michel Servet, 1211 Geneva 4, Switzerland.

E-mail: pmhawech1@yahoo.com

Received 17 May 2004; revised 22 June 2004; accepted 24 June 2004; published online 16 July 2004 sequestration of CDC2 in the cytoplasm, 14-3-3 $\sigma$ mediates a G2/M arrest. Genetic inactivation of 14-3-3 $\sigma$ was used to demonstrate its requirement for a stable arrest after DNA damage. Furthermore, 14-3-3 $\sigma$ may directly interact with and stabilize p53 suggesting a positive feedback loop. ${ }^{5}$ In addition to cell cycle regulation, $14-3-3 \sigma$ proteins may play a role in controlling apoptotic cell death. Through interaction with proapoptotic proteins BAD and BAX, 14-3-3 $\sigma$ has been shown to inhibit apoptosis.

Compelling evidence suggest that $14-3-3 \sigma$ is a tumor suppressor gene that contributes to cancer development. First, DNA damage leads to upregulation of $14-3-3 \sigma$ in colon cancer cells in a p53dependent manner. Second, gene inactivation of 14-3-3 $\sigma$ in cells leads to a defect in G2/M arrest normally induced by DNA damage. Third, irradiated 14-3-3 $\sigma-/$ - cells are not capable to stably arrest in G2 phase of the cycle to repair DNA damages leading to mitotic catastrophe and cell death. ${ }^{4}$ In breast epithelial cells, 14-3-3 $\sigma$ expression has been 
demonstrated to be post-translationally regulated through interaction with the estrogen responsive finger protein (EFP) leading to ubiquitinylation and rapid degradation. Furthermore, overexpression of EFP in vivo can induce tumors presumably by inducing genomic instability due to $14-3-3 \sigma$ loss. ${ }^{6}$ Finally, in skin epithelia, expression of $14-3-3 \sigma$ is upregulated upon differentiation in cells of the poststem cell compartment and immortalization of keratinocytes by experimental inactivation of 14-3-3 $\sigma$, underlines furthermore its role as tumor suppressor gene. ${ }^{7}$

Significant progress has been made in understanding the molecular genetics of urological and gynecological malignancies. There is strong experimental and clinical evidence that in addition to distinct genetic mutations, epigenetic mechanisms may play an important role in the pathogenesis of these tumors. ${ }^{8-10}$ Hypermethylation of CpG islands is a well-known epigenetic mechanism to inactivate a tumor suppressor gene. Silencing of gene expression by CpG hypermethylation has been shown to be an early event in cancer development, and in some cases may precede the neoplastic process. ${ }^{11,12}$ Likewise to other malignancies, silencing of an increasing number of genes by CpG island methylation has been demonstrated in renal, urothelial, ovarian, prostate and endometrial cancers. Best known examples are silencing of the VHL tumor-suppressor gene in renal carcinoma or loss of hormone receptor genes (estrogen, progesterone, androgen) in endometrial and prostate cancers respectively. ${ }^{13,14}$

Following the hypothesis of 14-3-3 $\sigma$ being a tumor suppressor gene, Ferguson et $a l^{15}$ analyzed 14-3-3 $\sigma$ expression in breast cancer. Downregulation of 14-3-3 $\sigma$ expression in tumor cell lines and primary tumor samples was associated with aberrant CpG island methylation. Following studies demonstrated epigenetic silencing of 14-3-3 $\sigma$ expression in gastric, liver, lung, cervical, oral squamous cell carcinoma, and basal cell carcinoma of the skin. ${ }^{16-21}$

The aims of this study were: first, to study the expression of $14-3-3 \sigma$ by using immunohistochemistry on a multiple tumor tissue microarrays containing a large number of urological and gynecological cancers. Second, to explore the relation between 14-3-3 $\sigma$ expression and p53 using the D07 and the PAB240 clone recognizing wild-type and mutated p53 isoforms respectively. Third, by focusing on adenocarcinoma of three organs such as prostate, endometrium and ovary, we intended to evaluate the relation between downregulation of 14-3-3 $\sigma$ and CpG island methylation in cell lines and primary tumor samples.

\section{Materials and methods}

\section{Case Selection and Multitumor Tissue Microarray}

A total of 350 cases of carcinomas arising in the urological and gynecological tracts has been selected from the surgical pathology archives. These malignancies include the following cases: urothelial bladder carcinoma $(n=45)$, squamous cell carcinoma of the uterine cervix $(n=9)$, endometrial carcinoma $(n=46)$, ovarian carcinoma $(n=21)$, breast carcinoma (ductal type $n=36$, lobular type $n=7$ ), renal carcinoma (clear cell type $n=60$, chromophobe $n=10$, papillary $n=16$ ), adenocarcinoma of the prostate $(n=40)$ and testicular tumors (seminoma $n=24$, embryonal $n=36$ ).

Paraffin-embedded tissues were used. The tissue microarray was constructed as described previously by Kononen et $\mathrm{al}^{22}$ After carefully choosing the morphologically representative region on the individual paraffin-embedded chosen blocks (donor blocks), a core tissue biopsy of $1.6 \mathrm{~mm}$ was punched and transferred to the donor paraffin-embedded block (receiver block). To overcome tumor heterogeneity, two punch biopsies were performed from different areas of each tumor. In total, 42 normal tissue from each of cervix $(n=2)$, breast $(n=10)$, kidney $(n=12)$, prostate $(n=10)$, and testis $(n=8)$ were also included in the array. owing to the difficulty of taking punch biopsy of normal urothelium for the array, whole sections of five normal bladder mucosa were studied separately for the expression of 14-3-3 $\sigma$. Sections of $4 \mu \mathrm{m}$ thickness were cut from the tumor microarray block and transferred to glass slides using the paraffin-sectioning aid system (adhesive-coated slides, adhesive tape, and UV lamp, Instrumedics Inc., Hockensack, NJ, USA). One section was stained by hematoxylineosin to evaluate the presence of the tumor.

\section{Immunohistochemical Staining Procedure}

Sections, of $4 \mu \mathrm{m}$ thickness, from formalin-fixed, paraffin-embedded tissue microarray were processed for immunohistochemistry. Endogenous peroxidase was blocked with $0.3 \%$ hydrogen peroxidase for $5 \mathrm{~min}$. Antigen retrieval was carried out in citrate buffer $(0.01 \mathrm{M}, \mathrm{pH} 6.0)$ at $98^{\circ} \mathrm{C}$ for $3 \mathrm{~min}$ in a pressure cooker. Then, sections were incubated with the 14-3-3 $\sigma$ (rabbit polyclonal, 1:50, IBL, Japan), p53/PAB240 (mouse monoclonal, 1:50, DAKO, Copenhagen, Denmark), and p53/D07 (mouse monoclonal, 1:400, DAKO) antibodies at room temperature. A subsequent reaction was performed with biotin-free HRP enzyme labeled polymer of Envision plus detection system (DAKO). Diaminobenzidine complex was used as chromogen. Evaluation of the immunohistochemistry was evaluated semiquantitatively by two pathologists (PM and MA) using double-head microscope, who were not aware of the original histological diagnosis. For p53, the cutoff of $10 \%$ of cells with positive nuclei staining pattern was considered as positive. As for 14-3-3 $\sigma$, staining was detected in the cytoplasm with sometimes nuclear staining. The cutoff of $50 \%$ positive cells was considered as positive. 


\section{Methylation-Specific PCR}

Genomic DNA was isolated from 66 formaldehydefixed and paraffin-embedded cases (17 ovarian, 30 prostate, 19 endometrial tumors) by using standard techniques. For molecular analysis tumor regions were mechanically separated from normal tissue if necessary, to have over $80 \%$ tumor cells in a given sample. Bisulfite treatment and PCR amplification was performed as previously described with some modification. ${ }^{15}$ In brief, $2 \mu \mathrm{g}$ genomic DNA was denatured in $\mathrm{NaOH}(0.2 \mathrm{~N})$ for $15 \mathrm{~min}$ at $37^{\circ} \mathrm{C}$ before adding $30 \mu \mathrm{l}$ hydrochinone ( $20 \mu \mathrm{M}$, Sigma) and $520 \mu \mathrm{l}$ of sodium bisulfite ( $3 \mathrm{M}, \mathrm{pH}$ 5.0). The samples were mixed and incubated at $50^{\circ} \mathrm{C}$ for $16 \mathrm{~h}$. Modified DNA was purified with the QIAGEN PCR purification kit, following the manufacturer's protocol (QIAGEN, Hildesheim, Germany). To complete the conversion, $6.3 \mu \mathrm{l}$ $\mathrm{NaOH}(5 \mathrm{~N})$ was added to the eluted DNA $(100 \mu \mathrm{l})$ and the samples incubated for $5 \mathrm{~min}$ at room temperature, followed by ethanol precipitation. DNA samples were then stored at $-20^{\circ} \mathrm{C}$ until further use. High-performance Taq polymerase (AmpliTaq-GOLD, Applied Biosystems, Rotkreuz, Switzerland) was used for all PCR reactions. All samples were first analyzed for amplification of the wild-type (WT) 14-3-3 $\sigma$ locus using the primers (WT-Forward: $5^{\prime}$-tat gag gac atg gca gcc ttc a-3' and WT-Reverse: $5^{\prime}$-cc cct cca ggc agc cct ctg g-3') recognizing a $138 \mathrm{bp}$ genomic DNA fragment (GenBank Acc. Do. NM_006142, position 210348). For methylation-specific PCR previously reported primer sets covering $\mathrm{CpG}$ dinucleotides numbers 3, 4, 8, and 9 of $14-3-3 \sigma$ were used. ${ }^{15}$ For each PCR reaction, three controls were included: in addition to a water control (negative), two DNA samples of breast cancer cell lines (MDA-MB-435, MCF-7) were analyzed known to result in strong signals for the methylated and unmethylated alleles, respectively (MCF-7, MDA-MB-435). ${ }^{15,20}$

\section{4-3-3 $\sigma$ Methylation and Messenger RNA Expression upon 5-Azacytidine Treatment}

Nine urological and gynecological cancer cell lines (all from the American Tissue Culture Collection (ATCC) cultivated according to the supplier's recommendations) were analyzed for $14-3-3 \sigma$ promoter methylation by PCR (as describe above). Two cancer cell lines (AN3CA, LNCAP) with strong 14-3-3 $\sigma$ CpG methylation were further analyzed for 14-3-3 $\sigma$ expression at the messenger RNA (mRNA) level before and after blocking cellular methylation by 5-azacytidine (5-Aza, SIGMA Biochemicals, Buchs, Switzerland) treatment as described previously. ${ }^{15}$ Cells were treated for three consecutive days with 5-Aza at concentrations ranging from $0.1-10 \mu \mathrm{M}$. A fresh stock solution was prepared each day $(0.5 \mathrm{mM}$ in medium) immediately before adding to the cells. $14-3-3 \sigma$ promoter methylation was checked by PCR before and after 5-Aza treatment. For quantitative mRNA expression analysis, total RNA was isolated using the Trizol $^{\mathrm{TM}}$ reagent following the manufacturer's instruction (Invitrogen Life Technologies, Carlsbad, CA, USA). Obtained total RNA was quantified by optical densitometry and $0.5 \mu \mathrm{g}$ were directly transcribed into first-strand complementary DNA (cDNA) using the first strand cDNA synthesis kit from Roche Molecular Biochemicals (Rotkreuz, Switzerland). To quantify the levels of 14-3-3 $\sigma$ mRNA levels $1 \mu \mathrm{l}$ of a $100 \times$ dilution of the cDNA $(20 \mu \mathrm{l})$ was used to proceed real-time PCR in iQTM SYBR Green Supermix (Biorad Laboratories Inc., Hercules, CA, USA). mRNA expression was evaluated by quantitative reverse transcriptase polymerase chain reaction (RT-PCR) analysis using an Icyler ${ }^{\mathrm{TM}}$ (Biorad). For each sample, PCR reactions were performed in triplicate. The following primers were used: sigma-G1 (forward primer) $5^{\prime}$-cca ggc tac ttc tcc cct c-3' and sigma-G2 (reverse primer) $5^{\prime}$-ctg tcc agt tct cag cca ca-3' resulting in a $99 \mathrm{bp}$ fragment (GenBank Acc. Do. NM_006142; position 11651263). The specificity of the PCR reaction was further checked by sequence analysis of the product appearing as single band of expected size on a $2.5 \%$ agarose gel. Expression levels of 14-3-3 $\sigma$ mRNA were normalized to the endogenous mRNA levels of GAPDH gene ( $\triangle \mathrm{CT}$ method).

\section{Statistical Analysis}

The associations between protein expressions were determined using Fisher's exact test. Differences in the proportion of protein expression among organs and tumor types were assessed with Fisher's exact test. All analysis was performed using the Stata 8.2, Statistical Package (College Station, TX, USA).

\section{Results}

\section{Distribution of 14-3-3 $\sigma$ Expression in Normal Urological and Gynecological Tissues}

The distribution of $14-3-3 \sigma$ protein expression in normal tissue was as follows: positive immunostaining was observed in the urothelial epithelium, periductal and periglandular cells of the prostate (Figure 1a,b) and breast and some glandular and ductal cells, and squamous epithelium of the exocervix of the uterus. On the other hand, no 14-3-3 $\sigma$ expression was seen in the germinal cells of the testis and in the ovary (follicules and stroma). There was very sporadic expression in renal tubules, but none in the glomeruli. There was very weak expression in the endometrial glands and endocervical glands. 

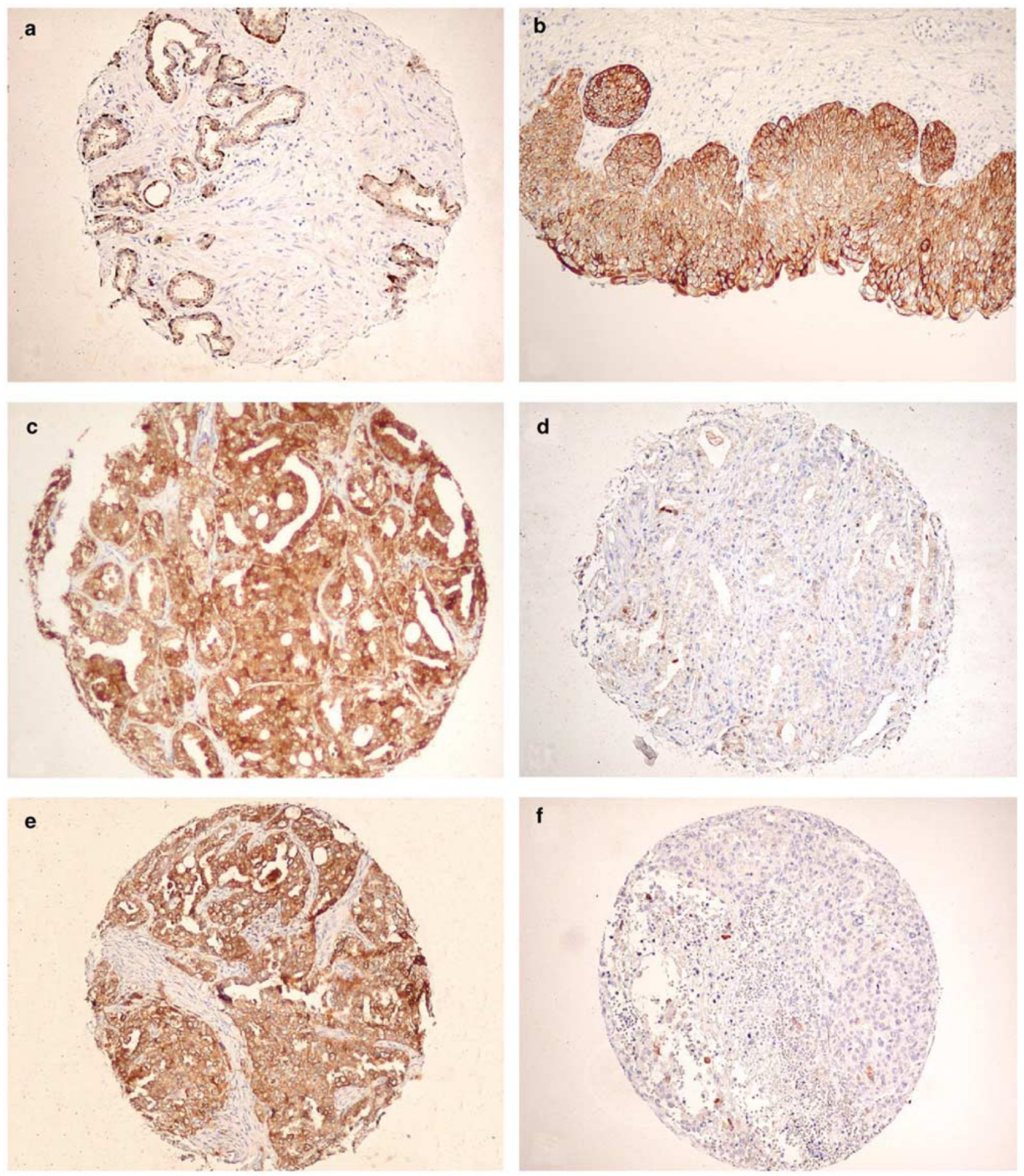

Figure 1 14-3-3 $\sigma$ protein expression in normal and malignant urological and gynecological tissues assessed by immunohistochemistry. (a) Normal prostate tissue stained with 14-3-3 $\sigma$ showed positivity of the basement membrane and some epithelial cells. (b) Normal bladder stained with 14-3-3 $\sigma$ showed positive staining of the cytoplasm of urothelial cells. (c) A case of prostatic adenocarcinoma sigma showing strong 14-3-3 $\sigma$ positivity in the cytoplasm and nuclei of tumor cells. (d) Prostatic adenocarcinoma negative for 14-3-3 $\sigma$. (e) A case of endometrial adenocarcinoma showing strong positivity of 14-3-3 $\sigma$. The expression by tumor cells was mainly cytoplasmic. (f) Endometrial adenocarcinoma negative for $14-3-3 \sigma$. 
Expression of 14-3-3 $\sigma$ in Urological and Gynecological Tumor Tissues

As summarized in Table 1, 14-3-3 $\sigma$ protein expression was found in $141(40.3 \%)$. of 350 overall tumors. The protein was expressed in 44 (98\%) urothelial bladder cancer, 22 (55\%) prostatic adenocarcinoma, six $(66.7 \%)$ squamous cell carcinoma of the cervix, 26 (56.6\%) endometrial adenocarcinoma, seven $(33 \%)$ ovarian adenocarcinoma and 10 (23\%) breast carcinoma. Similar to normal tissue, sporadic expression of $14-3-3 \sigma$ was detected in $10(11.7 \%)$ of renal tumors and $16(26.7 \%)$ of testicular tumors (Figure $1 \mathrm{c}-\mathrm{f}$ ).

Since 14-3-3 $\sigma$ has been shown to be regulated by p53, we next analyzed the tumors by using two antibodies recognizing p53, D07 recognizing an epitope in the amino (N)-terminus of the wild-type human p53 protein, and PAb240 recognizing only the mutant forms of p53. The p53 D07 was expressed in 78 cases $(22.4 \%)$ and negative in 270 cases $(77.6 \%)$. Signals for mutated $p 53$ were seen in 57 cases $(16.5 \%)$ and negative in 290 cases $(83.6 \%)$. There was no significant association between 14-3-3 $\sigma$ and p53 expression as detected by the p240 or the DO7 antibody $(p=0.9)$. Furthermore, and as expected, a significant association between p53 D07 and mutated p53 $(p<0.001)$ was observed.

\section{Downregulation of 14-3-3 $\sigma$ Expression in Prostate, Endometrial and Ovarial Carcinomas is Associated with 14-3-3 $\sigma$ CpG Methylation}

As summarized in Figure 2 and Table 2, methylation-specific PCR (MSP) analysis revealed signals for 14-3-3 $\sigma$ CpG island methylation in most of the cases analyzed. There was a significant association of CpG methylation with a weak or absent $14-3-3 \sigma$ protein expression in the 66 cases chosen from the tissue microarray (Table 2). All cases without sign of methylation (weak or absent amplification of the

Table 1 Expression of 14-3-3 $\sigma$, wild-type $p 53$ and mutated p53 in different tumor tissues of the urological and gynecological tracts

\begin{tabular}{|c|c|c|c|c|c|c|c|}
\hline & \multirow[t]{2}{*}{ Total number } & \multicolumn{2}{|c|}{$14-3-3 \sigma$} & \multicolumn{2}{|c|}{ p53 (DO7) } & \multicolumn{2}{|c|}{ p53 (pAb240) } \\
\hline & & Positive (n) & $\%$ & Positive (n) & $\%$ & Positive (n) & $\%$ \\
\hline Urothelial bladder carcinoma & 45 & 44 & 98 & 19 & 42.2 & 10 & 22.2 \\
\hline Prostatic adenocarcinoma & 40 & 22 & 55 & 1 & 2.5 & 1 & 2.5 \\
\hline Squamous cell carcinoma of the cervix & 9 & 6 & 66.7 & 1 & 11.1 & & 0 \\
\hline Endometrial adenocarcinoma & 46 & 26 & 56.6 & 9 & 19.6 & 3 & 6.5 \\
\hline Breast carcinoma & 43 & 10 & 23 & 4 & 9.3 & 4 & 9.3 \\
\hline Ductal type & 36 & & & & & & \\
\hline Lobular type & 7 & & & & & & \\
\hline Ovarian adenocarcinoma & 21 & 7 & 33 & 16 & 76.2 & 13 & 62 \\
\hline Renal tumors & 86 & 10 & 11.7 & 6 & 7 & 0 & 0 \\
\hline Renal cell carcinoma & 60 & & & & & & \\
\hline Chromophobe carcinoma & 10 & & & & & & \\
\hline Papillary carcinoma & 16 & & & & & & \\
\hline Testicular tumors & 60 & 16 & 26.7 & 22 & 38 & 26 & 45.6 \\
\hline Seminoma & 24 & & & & & & \\
\hline Embryonal carcinoma & 36 & & & & & & \\
\hline
\end{tabular}

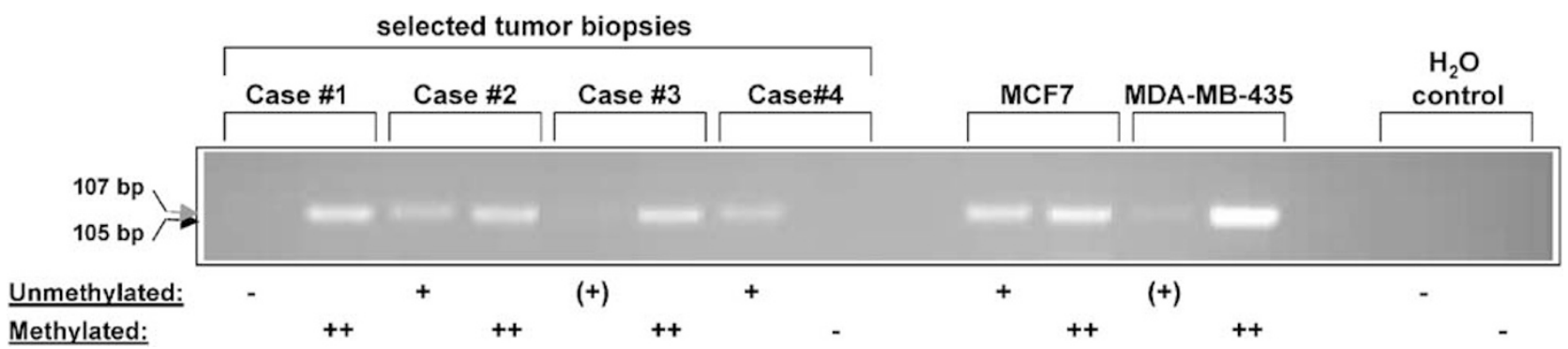

Figure 2 14-3-3 $\sigma$ methylation-specific PCR analysis in selected tumor biopsies. Methylation-specific PCR analysis showed cases with strong 14-3-3 $\sigma$ methylation and absence of unmethylated allele, cases with signals for unmethylated and methylated alleles of variable intensity $[(+),+,++]$ and cases with no signs of methylation. In each PCR reaction, in addition to a water negative control, DNA from breast carcinoma cells (MCF-7, MDA-MB-435) showing predominant signals for the unmethlylated and methylated alleles, respectively, were used. PCR products with close molecular weight (methylated allele, $105 \mathrm{bp}$; unmethylated allele, $107 \mathrm{bp}$ ) are marked with arrows. 
Table 2 Association of 14-3-3 $\sigma$ CpG island methylation (by methyaltion-specific PCR) and protein expression in 66 tumors from the urological and gynecological tracts

\begin{tabular}{|c|c|c|c|c|c|}
\hline & \multicolumn{5}{|c|}{ Methylation-specific PCR } \\
\hline & - & $(+)$ & + & ++ & \\
\hline \multicolumn{6}{|l|}{ M (methylated) } \\
\hline IHC-positive $(n=32)^{\mathrm{a}}$ & 2 & 15 & 14 & 1 & \\
\hline IHC-negative $(n=34)^{\mathrm{b}}$ & 0 & 6 & 19 & 9 & $P=0.003^{*}$ \\
\hline \multicolumn{6}{|l|}{$U$ (unmethylated) } \\
\hline IHC-positive $(n=32)$ & 1 & 13 & 14 & 4 & $P=0.006^{* *}$ \\
\hline IHC-negative $(n=34)$ & 9 & 16 & 9 & 0 & \\
\hline
\end{tabular}

Association between IHC-negativity and methylation signal (M)* and IHC-positivity and unmethylation signal (U)** .

${ }^{\mathrm{a}}$ IHC-positive cases (ovarian $(n=7)$, prostate $(n=15)$, endometrial $\left.(n=10)\right)$.

bIHC-negative (ovarian $(n=10)$, prostate $(n=15)$ and endometrial $(n=9)$ ).

Table 3 14-3-3 $\sigma$ CpG island methylation in urological and gynecological cancer cell lines

\begin{tabular}{llcc}
\hline Cell line & Origin & Unmethylated $^{\text {a }}$ & Methylated $^{\text {a }}$ \\
\hline DU-145 & Prostate CA & ++ & - \\
PC-3 & Prostate CA & ++ & - \\
LNcaP & Metastatic prostate CA & - & + \\
OVCA-429 & Ovarian CA & ++ & + \\
OVCAR-433 & Ovarian CA & + & + \\
OVCAR-3 & Ovarian CA & + & + \\
HOC-1B & Ovarian CA & - & + \\
AN3CA & Endometrial CA & ++ & + \\
HEC-59 & Endometrial CA & ++ \\
\hline
\end{tabular}

${ }^{\mathrm{a}}$ Signal strength in methylation-specfic PCR (MSP) analysis (see Figure 2).

methylated allele) showed normal expression of 14-3-3 $\sigma$ immunohistochemical staining $(p=0.006)$. In contrast, almost all cases showing evidence for strong methylation (weak or absent amplification of the unmethylated allele) showed only faint or absent 14-3-3 $\sigma$ protein expression $(p=0.003)$. These results suggest that $\mathrm{CpG}$ methylation of $14-3-3 \sigma$ may be responsible for downregulation of $14-3-3 \sigma$ expression in urological and gynecological cancers.

To further support the hypothesis that $\mathrm{CpG}$ promoter methylation leads to down-regulation of 14-3-3 $\sigma$ expression in urological and gynecological cancers, we analyzed a panel of established cells lines from ovarian cancer (OVCA-429, OVCAR-3, OVCAR-433, HOC-1B), prostate cancer (LNCAP, DU-145, PC-3) and endometrial cancer (HEC-59, AN3CA) by methylation-specific PCR. As shown in Table 3, five out of nine cell lines showed some 14$3-3 \sigma$ promoter methylation. In three of them, no signal for unmethylated alleles could be observed. We next ask whether demethylation by the DNA methyltransferase inhibitor 5-aza-2'-deoxycytidine (5-Aza) may be able to trigger re-expression of 14-3-3 $\sigma$ in cells with strong CpG island methylation. As shown in Figure 3a an increased signal for the unmethylated allele is observed after 3 days of 5-Aza treatment of LNCAP (prostate cancer) and AN3CA (endometrial cancer) cells. However, it is interesting to note that the sensitivity of both cell lines differs by factor 10 , with optimal signals after 1 and $10 \mu \mathrm{M}$ 5-Aza treatment. Further increase of the 5-Aza dose results in nonspecific toxicity without any significant demethylation of 14-3-3 $\sigma$ (data not shown). To analyze the link of $14-3-3 \sigma$ promoter methylation and gene expression, 14-3-3 $\sigma$ mRNA levels were further determined in both cell lines before and after 5 -Aza treatment. As shown in Figure $3 \mathrm{~b}$, dosedependent increase in 14-3-3 $\sigma$ mRNA can be observed in LNCAP and AN3CA cells upon demethylation by 5-Aza treatment. These findings show that downregulation of $14-3-3 \sigma$ in urological and gynecological cancers is a consequence of promoter $\mathrm{CpG}$ island methylation and suggest that pharmacological redirection of 14-3-3 $\sigma$ using smallmolecule DNA methyltransferase inhibitors may be of benefit in therapy of these cancers.

\section{Discussion}

In normal tissue, the $14-3-3 \sigma$ protein was found to be expressed in the urothelial epithelium, periductal and periglandular cells of the prostate and breast and some glandular and ductal cells, the exocervix 


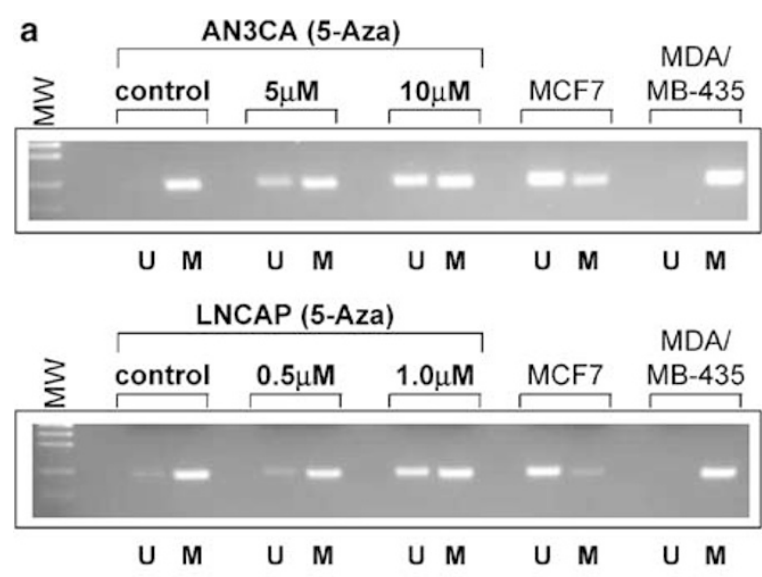

b
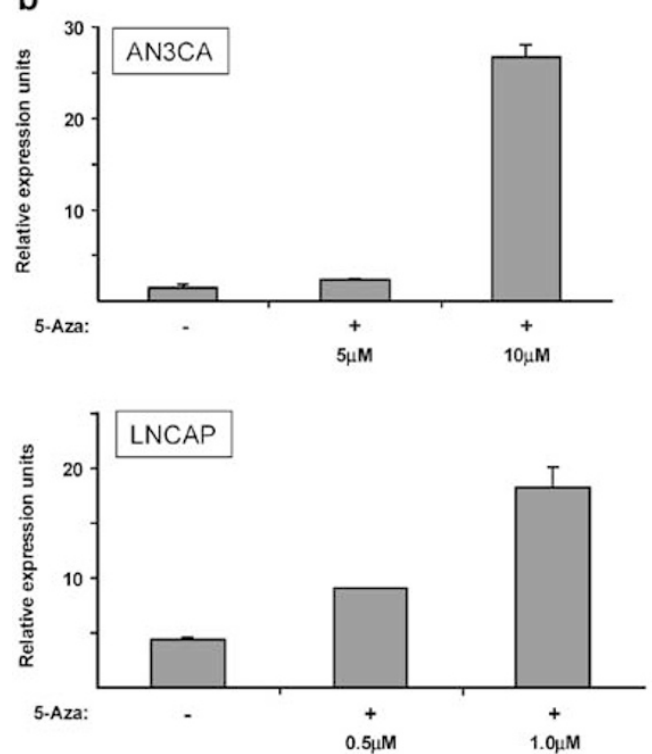

Figure 3 5-Aza treatment increases 14-3-3 $\sigma$ mRNA expression in selected cancer cell lines from prostate, ovary and endometrium. (a) AN3CA and LNCAP cells were treated for 3 days with 5-Aza, $0.1-10 \mu \mathrm{M})$ with renewal of the medium each day. Untreated AN3CA and LNCAP cells show an almost complete methylation of the 14-3-3 $\sigma$ promoter region. Upon 5-Aza treatment a dosedependent signal increase for unmethylated 14-3-3 $\sigma$ alleles can be observed. (b) 14-3-3 $\sigma$ mRNA levels were compared upon treatment of AN3CA (top panel) and LNCAP (bottom panel) cells with 5-Aza (0.1-10 $\mu \mathrm{M}, 3$ days) by real-time PCR. Relative levels were normalized to the GAPDH housekeeping gene mRNA expression levels. A dose-dependent increase in 14-3-3 $\sigma$ mRNA expression after 5-Aza treatment can be seen in both cell lines.

of the uterus, and its absence in the ovarian stroma, kidney, and testis. These results are very similar to those seen by Nakajima et al. ${ }^{23}$

In tumor tissues, $14-3-3 \sigma$ was more frequently expressed in urothelial bladder cancer, squamous cell carcinoma of the exocervix, followed by prostatic and endometrial adenocarcinomas, and it is the least expressed in carcinomas of the breast and ovary and in renal and testicular tumors. By performing PCR analysis for CpG island methylation, we showed that decrease 14-3-3 $\sigma$ expression is associated with methylation-dependent transcriptional silencing. Although we have not analyzed our cases for 14-3-3 $\sigma$ mutations, previous works analyzing a large number of cases from various tissues have failed to detect any mutation that may be responsible to downregulate 14-3-3 $\sigma$ expression. Including our series, epigenetic transcriptional silencing of $14-3-3 \sigma$ has been demonstrated in malignancies from prostate, endometrium, ovary, breast, lung, liver, skin, stomach and oral squamous cell carcinoma. ${ }^{15-18,20,21,24-26}$ Another recent immunohistochemical study has shown loss of $14-3-3 \sigma$ expression in prostate cancer. ${ }^{27}$ By preselecting areas of highest Gleason score in their tumor biopsies and by using a different 14-3-3 $\sigma$ antibody, these authors find low or absent levels of $14-3-3 \sigma$ in an even higher percentage of tumors.

Several studies have documented 14-3-3 $\sigma$ CpG methylation in dysplastic premalignant lesions or normal tissue adjacent to the tumor, suggesting that this process may represent an early event in carcinogenesis. ${ }^{20,24}$ However, in contrast to most malignancies, gene expression profiling as well as tissue microarray screens have recently demonstrated overexpression of $14-3-3 \sigma$ in pancreatic ductal adenocarcinomas and biliary cancers. ${ }^{28,29}$ Interestingly, deregulated overexpression of 14-3-3 $\sigma$ was associated with $\mathrm{CpG}$ island hypomethylation. Close association of $\mathrm{CpG}$ island hypomethylation with overexpression of multiple genes indicates that gene hypomethylation is a frequent epigenetic event in pancreatic cancer. ${ }^{30}$

Normal regulation of 14-3-3 $\sigma$ seems to be cell-type dependent since in contrast to the tissues listed above, 14-3-3 $\sigma$ seems to be regulated by constitutive CpG island methylation in lymphoid cells. Using MSP, Bhatia et $a l^{3}$ have shown that normal and malignant lymphoid cells constitutively express low levels of $14-3-3 \sigma$ associated with CpG methylation. ${ }^{31}$ This finding is of importance and has to be kept in mind when analyzing tumor samples for 14-3-3 $\sigma$ methylation. Strong inflammatory reactions within the tumor samples may lead to false-positive results. This phenomenon may also explain methylationspecific PCR signals in some cases of our series with normal $14-3-3 \sigma$ as determined by immunohistochemistry.

Whether $14-3-3 \sigma$ may play a role in renal or testicular malignancies is not completely understood. Sporadic and low levels of 14-3-3 $\sigma$ expression detected in our series of normal and malignant renal and testicular tissues suggest that 14-3-3 $\sigma$ may not play an important role in the pathogenesis of these lesions.

14-3-3 $\sigma$ has been identified as a p53-regulated inhibitor of $\mathrm{G} 2 / \mathrm{M}$ cell cycle progression. DNA damage induced by irradiation or cytotoxic agents lead to a time and dose-dependent upregulation of 14-3-3 $\sigma$ expression. ${ }^{4}$ Induced by DNA damage, stress and various oncogenes p53 is the central regulator of the cells apoptotic machinery. ${ }^{32}$ 
Inactivation of p53 in a large percentage of human cancers promotes checkpoint defects, cellular immortalization, genomic instability and inappropriate survival. Since p53 inactivation by mutation of interaction with viral proteins is a frequent event in urological and gynecological tumors, we compared expression of p53 with 14-3-3 $\sigma$ expression in our large series. ${ }^{33}$ Keeping in mind that p53/PAB240 detects about $80 \%$ of p53 mutations, in the present study the immunohistochemical status of p53 did not correlate with 14-3-3 $\sigma$ expression. In our tumors, we did not analyze the p53 mutation but previous studies showed that no correlation between genomic alterations of p53 and 14-3-3 $\sigma$ expression was observed in various cancer cell lines, and in primary vulval squamous cancer and primary neuroendocrine tumors. ${ }^{16,25,26}$ Like $p 53$, its homologs $p 63$ and $p 73$ can trigger expression of $14-3-3 \sigma$. In addition, repression of 14-3-3 $\sigma$ expression was seen by a tumor-associated p63 splice variant (Delta Np63) in vitro. ${ }^{34,35}$ However, again no correlation was found between the p63 status and 14-3-3 $\sigma$ expression in hyperproliferative skin disease. ${ }^{20}$ These studies all suggest independence of 14-3-3 $\sigma$ expression of p53 and its homologues p63 and p73.

Interestingly, there is evidence that $14-3-3 \sigma$ expression is also regulated at the post-transcriptional level. Cellular levels of 14-3-3 proteins including the sigma isoform have been recently shown to be regulated by tuberous sclerosis gene products hamartin and tuberin, suggesting that deregulation of 14-3-3 proteins might contribute to tumor formation in tuberous sclerosis patients. ${ }^{36} \mathrm{In}$ addition, in breast cancer cells $14-3-3 \sigma$ has been shown to undergo proteolysis mediated by the $E F P$. The EFP is a target gene of the estrogen receptor acting as an ubiquitin ligase of $14-3-3 \sigma .^{6}$ Whether EFP, widely expressed in hormone-sensitive tissues like uterus, ovary and mammary glands may play a role in $14-3-3 \sigma$ regulation of urological and gynecological cancers is subject of ongoing studies.

In summary, $14-3-3 \sigma$ is downregulated in a significant fraction of urological and gynecological tumors. In addition, we showed an association between 14-3-3 $\sigma$ CpG methylation and low level of expression, indicating that epigenetic silencing of 14-3-3 $\sigma$ may present a frequent aberration in ovarian, endometrial and prostatic adenocarcinomas.

\section{Acknowledgements}

We thank Mr L Métral and the histopathology laboratory especially Mrs D Fontana for her help. This study was in part supported by grants from the Swiss National Science Foundation and ONCOSUISSE to JS.

\section{References}

1 Hermeking H. The 14-3-3 cancer connection. Nat Rev Cancer 2003;3:931-943.

2 Yaffe MB. How do 14-3-3 proteins work?-Gatekeeper phosphorylation and the molecular anvil hypothesis. FEBS Lett 2002;513:53-57.

3 Tzivion G, Avruch J. 14-3-3 proteins: active cofactors in cellular regulation by serine/threonine phosphorylation. J Biol Chem 2002;277:3061-3064.

4 Hermeking H, Lengauer C, Polyak K, et al. 14-3-3 sigma is a p53-regulated inhibitor of $\mathrm{G} 2 / \mathrm{M}$ progression. Mol Cell 1997;1:3-11.

5 Yang HY, Wen YY, Chen CH, et al. 14-3-3 sigma positively regulates p53 and suppresses tumor growth. Mol Cell Biol 2003;23:7096-7107.

6 Urano T, Saito T, Tsukui T, et al. Efp targets 14-3-3 sigma for proteolysis and promotes breast tumour growth. Nature 2002;417:871-875.

7 Pellegrini G, Dellambra E, Golisano O, et al. p63 identifies keratinocyte stem cells. Proc Natl Acad Sci USA 2001;98:3156-3161.

8 Wooster R, Weber BL. Breast and ovarian cancer. N Engl J Med 2003;348:2339-2347.

9 Visakorpi T. The molecular genetics of prostate cancer. Urology 2003;62:3-10.

10 Spandidos DA, Dokianakis DN, Kallergi G, et al. Molecular basis of gynecological cancer. Ann NY Acad Sci 2000;900:56-64.

11 Herman JG, Baylin SB. Gene silencing in cancer in association with promoter hypermethylation. N Engl J Med 2003;349:2042-2054.

12 Esteller M, Herman JG. Cancer as an epigenetic disease: DNA methylation and chromatin alterations in human tumours. J Pathol 2002;196:1-7.

13 Navari JR, Roland PY, Keh P, et al. Loss of estrogen receptor (ER) expression in endometrial tumors is not associated with de novo methylation of the $5^{\prime}$ end of the ER gene. Clin Cancer Res 2000;6:4026-4032.

14 Herman JG, Latif F, Weng Y, et al. Silencing of the VHL tumor-suppressor gene by DNA methylation in renal carcinoma. Proc Natl Acad Sci USA 1994;91: 9700-9704.

15 Ferguson AT, Evron E, Umbricht CB, et al. High frequency of hypermethylation at the 14-3-3 sigma locus leads to gene silencing in breast cancer. Proc Natl Acad Sci USA 2000;97:6049-6054.

16 Suzuki $\mathrm{H}$, Itoh $\mathrm{F}$, Toyota $\mathrm{M}$, et al. Inactivation of the 14-3-3sigma gene is associated with $5^{\prime} \mathrm{CpG}$ island hypermethylation in human cancers. Cancer Res 2000;60:4353-4357.

17 Iwata N, Yamamoto $\mathrm{H}$, Sasaki S, et al. Frequent hypermethylation of $\mathrm{CpG}$ islands and loss of expression of the 14-3-3 sigma gene in human hepatocellular carcinoma. Oncogene 2000;19:5298-5302.

18 Osada H, Tatematsu Y, Yatabe Y, et al. Frequent and histological type-specific inactivation of 14-3-3sigma in human lung cancers. Oncogene 2002;21:2418-2424.

19 Gasco M, Sullivan A, Repellin C, et al. Coincident inactivation of 14-3-3sigma and p16INK4a is an early event in vulval squamous neoplasia. Oncogene 2002; 21:1876-1881.

20 Lodygin D, Yazdi AS, Sander CA, et al. Analysis of 14-3-3sigma expression in hyperproliferative skin diseases reveals selective loss associated with CpGmethylation in basal cell carcinoma. Oncogene 2003; 22:5519-5524. 
21 Kang GH, Lee S, Kim WH, et al. Epstein-Barr viruspositive gastric carcinoma demonstrates frequent aberrant methylation of multiple genes and constitutes CpG island methylator phenotype-positive gastric carcinoma. Am J Pathol 2002;160:787-794.

22 Kononen J, Bubendorf L, Kallioniemi A, et al. Tissue microarrays for high-throughput molecular profiling of tumor specimens. Nat Med 1998;4:844-847.

23 Nakajima T, Shimooka H, Weixa $\mathrm{P}$, et al. Immunohistochemical demonstration of 14-3-3 sigma protein in normal human tissues and lung cancers, and the preponderance of its strong expression in epithelial cells of squamous cell lineage. Pathol Int 2003;53:353-360.

24 Umbricht CB, Evron E, Gabrielson E, et al. Hypermethylation of 14-3-3 sigma (stratifin) is an early event in breast cancer. Oncogene 2001;20:3348-3353.

25 Gasco M, Bell AK, Heath V, et al. Epigenetic inactivation of 14-3-3 sigma in oral carcinoma: association with p16(INK4a) silencing and human papillomavirus negativity. Cancer Res 2002;62:2072-2076.

26 Yatabe Y, Osada H, Tatematsu Y, et al. Decreased expression of 14-3-3sigma in neuroendocrine tumors is independent of origin and malignant potential. Oncogene 2002;21:8310-8319.

27 Cheng L, Pan CX, Zhang JT, et al. Loss of 14-3-3sigma in prostate cancer and its precursors. Clin Cancer Res 2004;10:3064-3068.

28 Logsdon CD, Simeone DM, Binkely C, et al. Molecular profiling of pancreatic adenocarcinoma and chronic pancreatitis identifies multiple genes differentially regulated in pancreatic cancer. Cancer Res 2003;63: 2649-2657.
29 Swierczynski SL, Maitra A, Abraham SC, et al. Analysis of novel tumor markers in pancreatic and biliary carcinomas using tissue microarrays. Hum Pathol 2004;35:357-366.

30 Sato N, Maitra A, Fukushima N, et al. Frequent hypomethylation of multiple genes overexpressed in pancreatic adenocarcinoma. Cancer Res 2003;63: 4158-4166.

31 Bhatia K, Siraj AK, Hussain A, et al. The tumor suppressor gene 14-3-3sigma is commonly methylated in normal and malignant lymphoid cells. Cancer Epidemiol Biomarkers Prev 2003;12: 165-169.

32 Fridman JS, Lowe SW. Control of apoptosis by $p 53$. Oncogene 2003;22:9030-9040.

33 Soussi T, Dehouche K, Beroud C. p53 website and analysis of p53 gene mutations in human cancer: forging a link between epidemiology and carcinogenesis. Hum Mutat 2000;15:105-113.

34 Westfall MD, Mays DJ, Sniezek JC, et al. The Delta Np63 alpha phosphoprotein binds the p21 and 14-3-3 sigma promoters in vivo and has transcriptional repressor activity that is reduced by Hay-Wells syndrome-derived mutations. Mol Cell Biol 2003; 23:2264-2276.

35 De Laurenzi V, Melino G. Evolution of functions within the p53/p63/p73 family. Ann NY Acad Sci 2000;926:90-100.

36 Hengstschlager M, Rosner M, Fountoulakis M, et al. Tuberous sclerosis genes regulate cellular 14-3-3 protein levels. Biochem Biophys Res Commun 2003; 312:676-683. 